\title{
Occurrence of nitric oxide synthase in Megoura viciae Buckton (Homoptera, Aphididae): an histochemical and immunohistochemical localisation
}

\author{
S. Ganassi, ${ }^{1}$ D. Tagliazucchi, ${ }^{2}$ L. Mola ${ }^{3}$ \\ ${ }^{1}$ Department of Animal Biology, University of Modena and Reggio Emilia, Modena; ${ }^{2}$ Department of \\ Agricultural Science, University of Modena and Reggio Emilia, Reggio Emilia; ${ }^{3}$ Department of \\ Paleobiology Museum and Botanical Garden, Section of Anatomical Museums, University of Modena and \\ Reggio Emilia, Modena, Italy
}

(C)2005, European Journal of Histochemistry

Nitric oxide (NO) is known to be involved in many physiological reactions of insects. We analysed NOS localisation in aphids of the species Megoura viciae by means of histochemical reaction for the NADPH-diaphorase activity and immunohistochemical methods for UNOS, nNOS and iNOS. The obtained data provided a complex and peculiar pattern of NOS distribution in cells and tissue of $M$. viciae. The histochemical reaction for NADPH-diaphorase was an indicative, but not exact marker of NOS localisation in aphids. The use of anti uNOS antiserum (frequently applied in insects) was of limited value in our specimens, whereas more satisfactory results were obtained with anti nNOS and iNOS antisera of human origin. The results of Western blot analysis confirmed the immunohistochemical ones, showing an aphid protein that reacted strongly with the polyclonal antibody anti-iNOS and anti-nNOS while a similar protein band was weakly immunoreactive with the polyclonal antibody anti-uNOS. Our results suggest that NO, prevalently synthesised by calcium/calmodulin-dependent isoform, plays important physiological roles both in adult and embryological stages of aphids. The data of principal interest was NOS presence in bacteriocytes, cells that host symbiotic prokaryotes belonging to the species Buchnera aphidicola, and in nuclei of adipocytes and gut cells.

Key words: insects, aphids, NADPH-diaphorase, nervous system, salivary glands, adipocytes, bacteriocytes.

Correspondence: L. Mola,

Dipartimento del Museo di Paleobiologia e dell'Orto Botanico, Comparto Musei Anatomici, Via Berengario n 14,

41100 Modena, Italy.

Tal: +39.059.2055535.

Fax: +39.059.2055548.

E-mail: mola.lucrezia@unimore.it

Paper accepted on September 23, 2005

European Journal of Histochemistry

2005; vol. 49 issue 4 (Oct-Dec): 385-393
$\mathrm{N}$ itric oxide (NO) is a membrane permeant signalling molecule which leads to the formation of cyclic GMP in target cells. The family of enzymes which form NO from L-arginine are known as nitric oxide synthases (NOS) and NO generation occurs in the presence of NADPH as cofactor. NOS isoforms were originally named after the tissue of origin, i.e. eNOS (endothelial NOS), nNOS (neuronal NOS) and iNOS (inducible, macrophage NOS). The eNOS and nNOS are constitutive calcium/calmodulin-dependent isoforms while iNOS is calcium/calmodulin-independent isoform one detected in different type of tissues.

A number of research provided strong evidence that NO is a signal molecule highly conserved during biological evolution. The cloning of a NOS homologue in Drosophila melanogaster (Regulski and Tully, 1995), Rhodnius prolixus (Yuda et al., 1996) Anopheles stephensi (Luckhart et al., 1998) Manduca sexta (Nighorn et al., 1998) and Bombix mori (Imamura et al., 2002) revealed that vertebrates and insects share the same identified NOS functional domain. In particular, the insect NOSs have higher amino acid sequence overlap with mammalian nNOS (mean of $49 \%$ identity) than iNOS ( $44 \%$ identity) or eNOS ( $47 \%$ identity) (Luckhart and Rosemberg, 1999). Analysis of NOS sequences in these insect species also reveals extensive regions of $100 \%$ identity in the catalytic domains and the co-factor binding sites for calmodulin, FAD, FMN and NADPH (Davies, 2000). Comparing available protein sequences for different phyla, insect NOS sequences form a distinct group, most closely related to human nNOS and eNOS, while iNOS family clusters in a separate group. The high degree of similarity at both the gene and protein level between insect NOS and nNOS over 400 million years of evolution suggests that the nNOS gene is the ancestor of the NOS gene family 